\title{
A potential acetyltransferase involved in Leishmania major metacaspase-dependent cell death
}

Louise Basmaciyan ${ }^{1}$, Nadine Azas ${ }^{2,3}$ and Magali Casanova $2,3^{*}$

\begin{abstract}
Background: Currently, there is no satisfactory treatment for leishmaniases, owing to the cost, mode of administration, side effects and to the increasing emergence of drug resistance. As a consequence, the proteins involved in Leishmania apoptosis seem a target of choice for the development of new therapeutic tools against these neglected tropical diseases. Indeed, Leishmania cell death, while phenotypically similar to mammalian apoptosis, is very peculiar, involving no homologue of the key mammalian apoptotic proteins such as caspases and death receptors. Furthermore, very few proteins involved in Leishmania apoptosis have been identified.

Results: We identified a protein involved in Leishmania apoptosis from a library of genes overexpressed during Leishmania differentiation during which autophagy occurs. Indeed, the gene was overexpressed when L. major cell death was induced by curcumin or miltefosine. Furthermore, its overexpression increased L. major curcumin- and miltefosine-induced apoptosis. This gene, named LmjF.22.0600, whose expression is dependent on the expression of the metacaspase, another apoptotic protein, encodes a putative acetyltransferase.

Conclusions: This new protein, identified as being involved in Leishmania apoptosis, will contribute to a better understanding of Leishmania death, which is needed owing to the absence of a satisfactory treatment against leishmaniases. It will also allow a better understanding of the original apoptotic pathways of eukaryotes in general, while evidence of the existence of such pathways is accumulating.
\end{abstract}

Keywords: Leishmania, Apoptosis, Autophagy, Therapeutic target

\section{Background}

Leishmania are flagellated protozoan parasites of the family Trypanosomatidae. They are responsible, in the mammalian host, for leishmaniases that are neglected tropical diseases threatening, according to the World Health Organization, between 700,000 and 1 million people worldwide and causing between 20,000 and 30,000 deaths each year. They are transmitted by the bite of a female sand fly where they accumulate in the gut in an extracellular flagellated form called the promastigote form. In the mammalian host, promastigotes are phagocytized by macrophages where they transform into an

\footnotetext{
*Correspondence: magali.casanova@univ-amu.fr

${ }^{2}$ Aix Marseille Univ, IRD, AP-HM, SSA, VITROME, Marseille, France

Full list of author information is available at the end of the article
}

intracellular immobile form with a much-reduced flagellum called the amastigote form. At present, there is no satisfactory treatment available for leishmaniases, owing to the cost and the parenteral mode of administration of the current drugs for people mainly living in developing countries, but also owing to the numerous side effects of many of these treatments and to the increasing emergence of drug resistance. As a consequence, new therapeutic tools are urgently required against leishmaniases. With this in mind, we decided to focus on Leishmania originalities, notably Leishmania cell death, with a view to designing new drugs targeting the parasite death in the future. Indeed, Leishmania cell death appears very peculiar: different stimuli can induce a form of cell death with the same phenotypic features as mammalian apoptosis (including cell shrinkage, chromatin condensation, DNA 
fragmentation and mitochondrial membrane depolarization) that we can call Leishmania apoptosis [1]. However, no key protein involved in the mammalian apoptosis, such as caspases or death receptors, could be identified in the parasite [2, 3]. Furthermore, very few proteins involved in Leishmania apoptosis have been identified, among which the metacaspase described as stimulating $L$. major apoptosis [4] or as a negative regulator of $L$. mexicana amastigote proliferation [5].

As part of this study, we identified a gene coding for a putative acetyltransferase, involved in L. major apoptosis, LmjF.22.0600. This gene is overexpressed under different pro-apoptotic conditions and its in vitro overexpression increases L. major curcumin- and miltefosine-induced apoptosis. Furthermore, the expression of the metacaspase is required for LmjF.22.0600 gene expression.

\section{Results}

\section{LmjF.22.0600 and its orthologues in different Leishmania} species

In Leishmania, numerous large-scale studies, involving transcriptomics and proteomics, have been conducted concerning the differentiation step from the vector promastigote form to the host cell amastigote form [6-12] during which cells undergo the cell survival process autophagy [13, 14]. Furthermore, there is a close link between apoptosis and autophagy, with accumulating evidence that, in addition to their mutual inhibition, one can activate the other. In particular, autophagy may induce apoptosis, by catabolizing parts of the cells after sequestration in autophagosomes and degradation in lysosomes, or by activating the apoptotic pathway [15]. As a consequence, we picked genes/proteins that were overexpressed during the differentiation stage and studied their involvement in Leishmania apoptosis. We focused on the LinJ22_V3.0470 gene which was 3.59 times more expressed under environmental conditions of promastigote to amastigote differentiation (temperature increase up to $37^{\circ} \mathrm{C}$ and $\mathrm{pH}$ decrease at 4.5 for 4 days) [6].

When comparing the protein sequence of different Leishmania species orthologues, we observed that $L$. braziliensis and $L$. infantum sequences contained an additional N-terminal domain of 24 amino-acids and L. mexicana and L. donovani, an additional $\mathrm{N}$-terminal domain of 60 amino-acids (Fig. 1a). However, the protein sequence has been highly preserved among Leishmania species, with identity percentages ranging from $66.7 \%$ (between L. braziliensis and L. major) to $99.8 \%$ (between $L$. donovani and $L$. infantum) (Fig. 1a, b). Not surprisingly, the cladogram gathers species inducing the same form of leishmaniasis: mucocutaneous leishmaniasis for L. braziliensis and L. mexicana, visceral leishmaniasis for $L$. donovani and L. infantum. Orthologues have also been found in parasites from the same family: Trypanosoma brucei (Tb.927.7.2530) and T. cruzi (TcCLB.511813.10).

The $L m j F .22 .0600$ gene is overexpressed during cell death induced by curcumin, miltefosine and pentamidine

We first measured the expression of the LmjF.22.0600 gene under pro-apoptotic conditions in log-phase promastigote $L$. major cells, in comparison to control conditions. For this, we carried out reverse-transcription quantitative PCR (RT-qPCR) on untreated cells and cells treated with curcumin, miltefosine or pentamidine. We chose these three drugs because they all induce L. major apoptosis [16] but through three different apoptotic pathways. Indeed, while curcumin and miltefosine respectively inhibit and activate the $L$. major metacaspase, pentamidine induces apoptosis in a metacaspase-independent manner [17]. We observed that the LmjF.22.0600 gene was significantly overexpressed under all three apoptotic conditions compared to the housekeeping control gene $k m p 11$ and untreated conditions. Indeed, with curcumin and miltefosine, the expression of LmjF.22.0600 normalized to the expression of $k m p 11$ was, on average, two times higher than the expression in untreated cells and it was 15 times higher in pentamidine-treated cells than in untreated cells (Fig. 2). This result indicates that LmjF.22.0600 expression is induced under pro-apoptotic conditions in L. major.

\section{Overexpression of $L m j F .22 .0600$ increases curcumin- and miltefosine-induced cell death}

To confirm the role of LmjF.22.0600 in L. major cell death, we overexpressed this gene by introducing it into the expression vector pTH6nGFPc and transfecting the $L$. major strain with this recombinant vector, which was maintained episomally in the cells. After the drug selection of the parasites overexpressing LmjF.22.0600, we verified that this overexpression did not induce any difference in cell growth compared to WT cells (see Additional file 1: Figure S1). We then studied the consequences of the overexpression under pro-apoptotic conditions. We first noted that the overexpression of LmjF.22.0600 induced a significant decrease in cell concentration when apoptosis was induced by the addition of curcumin or miltefosine (Fig. 3a). Conversely, during pentamidine-induced cell death, overexpression of the gene had no consequence on cell concentration (Fig. 3a).

We then carried out a methyl thiazol tetrazolium (MTT) assay, which allowed us to determine the inhibitory concentration $50 \%$ (IC50) of different drugs, that is to say the concentration of drug that inhibits $50 \%$ of cell growth in comparison to the control, for the WT strain and the strain overexpressing LmjF.22.0600. Figure $3 \mathrm{~b}$ shows that the IC50 of curcumin and miltefosine 


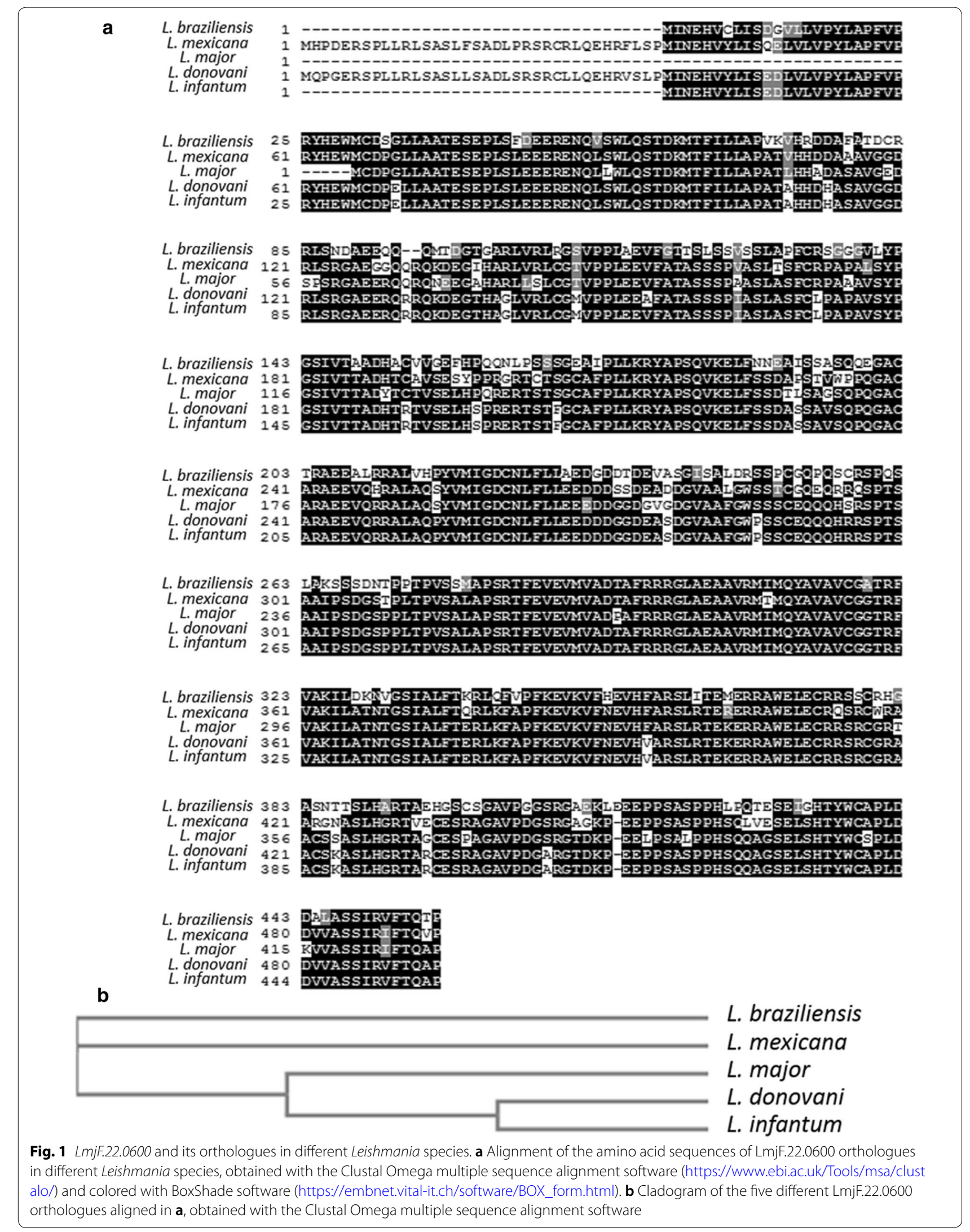




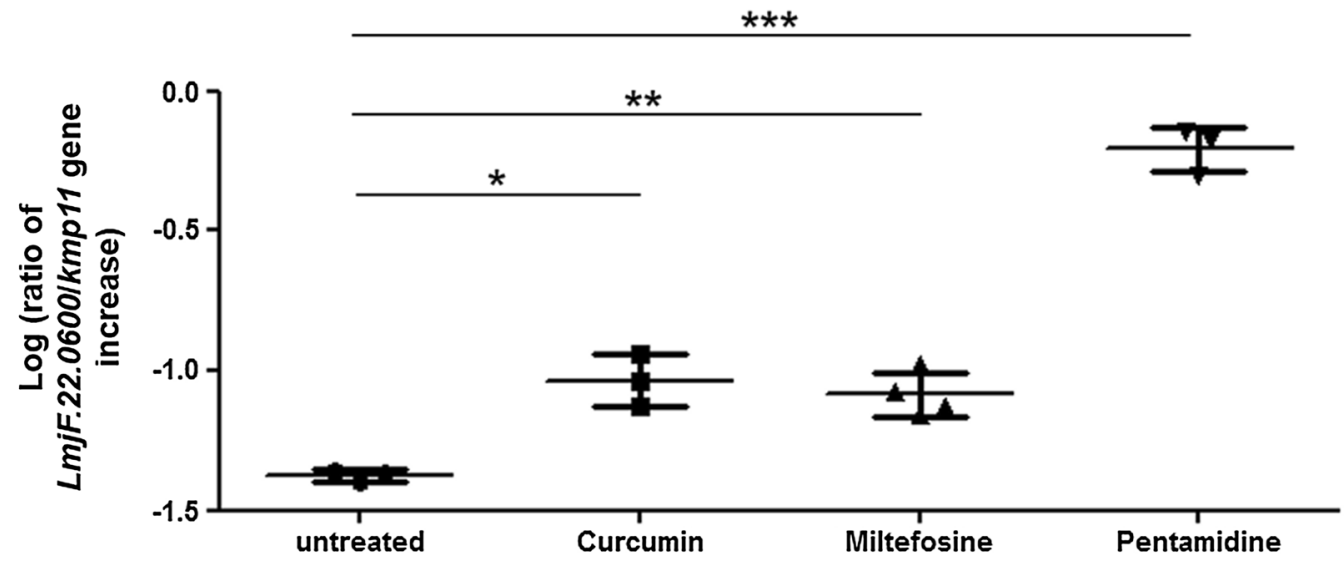

Fig. 2 The LmjF.22.0600 gene is overexpressed in different pro-apoptotic conditions. Ratio of expression of the LmjF.22.0600 gene to kmp 11 control gene, represented in log, as measured by RT-qPCR, in untreated cells and cells treated for $24 \mathrm{~h}$ with $50 \mu \mathrm{M}$ curcumin, $40 \mu \mathrm{M}$ miltefosine or 100 $\mu \mathrm{M}$ pentamidine. Mean from three independent experiments for curcumin and pentamidine, and four independent experiments for miltefosine. Unpaired t-test: ${ }^{*} P<0.05,{ }^{* *} P<0.01$ and ${ }^{* *} P<0.001$
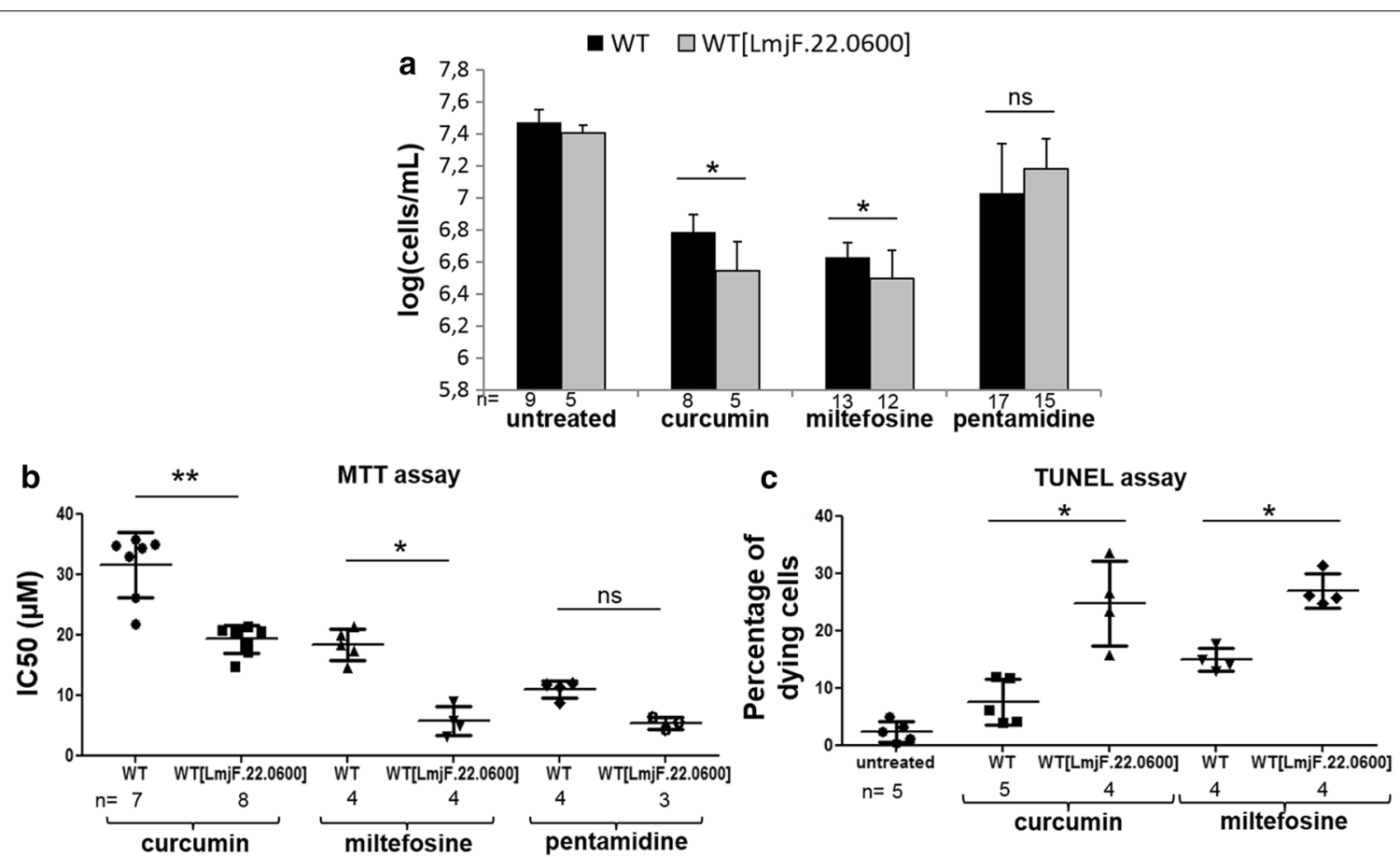

Fig. 3 The overexpression of LmjF.22.0600 increases curcumin- and miltefosine-induced cell death. a Cell concentration of WT cells and cells overexpressing LmjF.22.0600 [WT(LmjF.22.0600)] in untreated cells and cells treated with curcumin (50 $\mu \mathrm{M})$, miltefosine (40 $\mu \mathrm{M})$ and pentamidine $(50$ $\mu \mathrm{M})$, as measured with a hemocytometer. $\mathbf{b}$ IC50 values of curcumin, miltefosine and pentamidine in the WT strain and the strain overexpressing LmjF.22.0600, from MTT assays. c Percentage of dying cells, i.e. TUNEL-positive cells and cells without a nucleus, in the WT and LmjF.22.0600 overexpressing strains, when left untreated or treated with $50 \mu \mathrm{M}$ curcumin or $40 \mu \mathrm{M}$ miltefosine for $24 \mathrm{~h}$. Values are shown as the mean \pm SD. The number of independent experiments that were carried out is given below the bars. Unpaired Wilcoxon-Mann-Whitney test: ns, not significant, ${ }^{*} P<$ 0.05 and ${ }^{* *} P<0.01$

was significantly lower in the strain overexpressing LmjF.22.0600 than in the WT strain. Concerning pentamidine, no significant difference in the IC50 was observed between the WT and the recombinant strain (Fig. 3b). These results, from cell counting and IC50, 
show the involvement of LmjF.22.0600 in L. major curcumin- and miltefosine-induced cell death.

In order to clarify the type of cell death induced by curcumin and miltefosine when LmjF.22.0600 was overexpressed, we carried out a TUNEL assay to detect DNA fragmentation, a clear characteristics of apoptotic cells, even in the Leishmania parasite [16]. In this assay, we measured the percentage of dying cells, i.e. the percentage of cells with a TUNEL-positive nucleus and also of cells that no longer had a nucleus. The significant increase in the percentage of dying cells with curcumin and miltefosine, when LmjF.22.0600 was overexpressed, in comparison to WT cells, indicates that LmjF.22.0600 increased L. major curcumin- and miltefosine-induced apoptosis (Fig. 3c).

\section{The expression of $L m j F .22 .0600$ is inhibited} in the metacaspase-deleted strain

We measured by RT-qPCR the expression of LmjF.22.0600 in the WT strain and in the strain deleted from the $L$. major metacaspase LmjMCA. We observed that LmjF.22.0600 expression, normalized to the expression of the kmp11 control gene, was significantly inhibited in the LmjMCA-deleted strain (Fig. 4). On average, LmjF.22.0600 expression, normalized to kmp11 expression, was 13 times smaller in the LmjMCA-deleted strain than in the WT strain. Yet, the LmjF.22.0600 gene was present in the genome of both strains, as confirmed by PCR analysis (see Additional file 2: Figure S2). As a consequence, the expression of LmjMCA is required for expression of the $L m j .22 .0600$ gene.

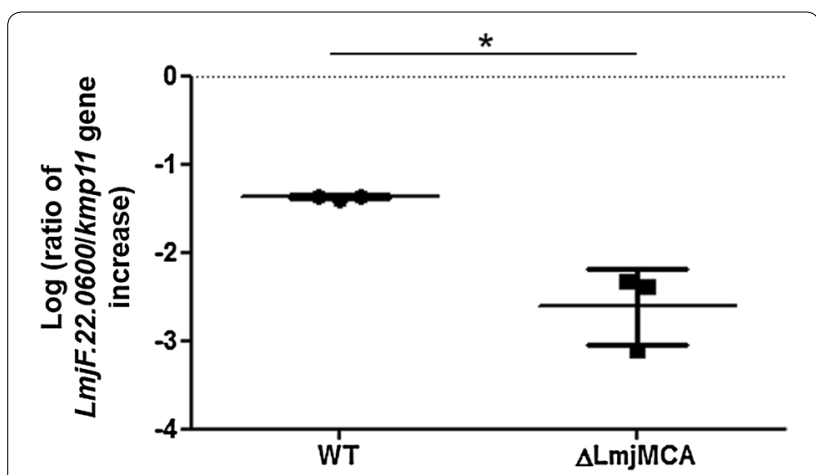

Fig. 4 The expression of $L m j F .22 .0600$ is inhibited in the metacaspase-deleted strain. RT-qPCR quantification of LmjF.22.0600 expression normalized to the expression of the control gene kmp11, in the WT and LmjMCA-deleted strains (logarithmic scale). Values are shown as the mean \pm SD from three independent experiments. Unpaired t-test: ${ }^{*} P<0.05$

\section{Discussion}

In this study, we identified a protein called LmjF.22.0600 involved in $L$. major apoptosis, the corresponding gene being overexpressed after the addition of different proapoptotic drugs and its overexpression increasing curcumin- and miltefosine-induced cell death. We can note that the inhibition of the expression of the gene by CRISPR/Cas 9 gave unexpected results. More precisely, while it induced a higher cell concentration compared to the WT cells after the addition of curcumin, it induced no significant difference in cell concentration after the addition of miltefosine (see Additional file 3: Figure S3a). Furthermore, co-staining with calcein and propidium iodide (PI), Leishmania apoptotic markers [18], did not allow us to detect any apoptosis inhibition or activation when the gene was deleted (see Additional file 3: Figure S3b, c). However, in Leishmania, cell death is essential for avoiding hyperparasitism by regulating the parasite cell density in the sand fly vector and in the mammalian host [19], for eliminating unfit cells and therefore allowing the fittest cells to survive [20], and for modulating host immunity, for instance by inducing the release of anti-inflammatory cytokines and the downregulation of pro-inflammatory cytokines, which would favor parasite survival [19]. Owing to the importance of apoptosis in the parasite, we can hypothesize that the absence of effect of LmjF.22.0600 deletion would be due to the involvement of other protein(s) that would take over in the apoptotic pathway. LmjF.22.0600 encodes a potential acetyltransferase that would induce posttranslational modifications participating in L. major apoptosis. The protein LmjF.22.0600 is involved in the same apoptotic pathway as the metacaspase LmjMCA. Indeed, the expression of the metacaspase is required for LmjF.22.0600 expression. Furthermore, LmjF.22.0600 overexpression had no consequence on pentamidineinduced L. major cell death, whereas pentamidine induces apoptosis through a metacaspase-independent pathway [17]. Our hypothesis is that the pro-apoptotic drug miltefosine induces the catalytic activation of LmjMCA, inducing LmjF.22.0600 gene expression activation, LmjF.22.0600 protein overexpression, and Leishmania apoptosis via proteins that are currently unknown. This constitutes the sixth protein identified as being involved in Leishmania apoptosis, with the metacaspase [4], the endonuclease $G[21,22]$, the cysteine proteinase $\mathrm{C}$ [23], the pro-apoptotic molecule with a $\mathrm{BH} 3$ domain Li-BH3AQP [24] and the hydrolase LmjHYD36 [25]. These six proteins involved in Leishmania apoptotic pathways are at variance with the idea of incidental death [3]. On the contrary, it is in concordance with the idea of programmed (physiological) or regulated (non physiological) cell death in this unicellular parasite. 
LmjF.22.0600 was identified from a library of genes overexpressed during the Leishmania promastigote to amastigote differentiation process during which autophagy occurs. As a consequence, while many experiments have been carried out on this step, it is an important source of genes/proteins potentially involved in Leishmania apoptosis. Through this approach, many genes other than LmjF.22.0600, coding proteins involved in Leishmania apoptosis, could therefore be identified, such as the recently published gene LmjF.36.6540 coding a potential hydrolase [25]. Here, the experiments have been carried out in vitro and on the promastigote $L$. major forms, using drugs as apoptotic inducers. Therefore, we can wonder whether the identified protein is also involved in vivo in Leishmania apoptosis within the sand fly gut or within the mammalian host.

\section{Conclusions}

The identification of additional genes involved in Leishmania apoptosis, even in vitro, contributes to a better understanding of original apoptotic pathways (notably caspase-independent) of eukaryotes in general, while evidence of the existence of such pathways is accumulating [26]. It also contributes to a better understanding of Leishmania biology, an essential element for the future design of new drugs to treat the neglected tropical diseases, leishmaniases, for which there are no satisfactory treatments.

\section{Methods}

\section{Parasites}

For L. major 'Friedlin' promastigotes (MHOM/IL/81/ Friedlin) culture, $10^{6}$ cells $/ \mathrm{ml}$ were routinely seeded in Schneider's Drosophila medium (Thermo Fisher Scientific, Waltham, MA, USA) supplemented with $100 \mathrm{U} / \mathrm{ml}$ penicillin, $100 \mu \mathrm{g} / \mathrm{ml}$ streptomycin, $2 \mathrm{mM}$ glutamine and $20 \%$ fetal calf serum (Thermo Fisher Scientific) at $26{ }^{\circ} \mathrm{C}$ for $48 \mathrm{~h}$ before subculturing when reaching $10^{7}$ cells $/ \mathrm{ml}$.

\section{Induction of cell death}

Logarithmic L. major cells were harvested by centrifugation and incubated at $10^{6}$ cells $/ \mathrm{ml}$ for the MTT assays or at $10^{7}$ cells $/ \mathrm{ml}$ for all other experiments, with 30 or 50 $\mu \mathrm{M}$ curcumin (Sigma-Aldrich, Saint-Louis, MO, USA), $40 \mu \mathrm{M}$ miltefosine (Santa Cruz Biotechnology, Dallas, TX, USA) or 50 or $100 \mu \mathrm{M}$ pentamidine (Sigma-Aldrich), drug concentrations previously shown as inducing $L$. major apoptosis [16].

\section{Reverse-transcription quantitative PCR}

For RNA extraction, the RNeasy Plus mini kit (Qiagen, Courtaboeuf, France) was used according to the manufacturer's procedure, the final RNA concentration being evaluated with a Nanodrop 2000c spectrophotometer (Thermo Fisher Scientific). One-step reverse transcription was performed using the high capacity cDNA reverse transcription kit (Applied Biosystems, Foster City, CA, USA) according to the manufacturer's instructions. For the quantitative PCR, $5 \mu \mathrm{l}$ of cDNA was added to $20 \mu \mathrm{l}$ of PCR mix containing Sybr Green I (Roche, Meylan, France) and placed in a Light Cycler 480 (Roche) with the following cycling conditions: Taq polymerase activation at $95{ }^{\circ} \mathrm{C}$ for 10 min then 45 cycles of amplification of $95{ }^{\circ} \mathrm{C}$ for $15 \mathrm{~s}$ and $60{ }^{\circ} \mathrm{C}$ for $60 \mathrm{~s}$. The $k m p 11$ (kinetoplastid membrane protein 11) gene was used as a control. Gene expression was calculated using the Pfaffl method in which the efficiency of each pair of primers is used, after its determination using the serial dilution method on the basis of a linear regression slope [27]. The oligonucleotides used in this study are listed in Additional file 4: Table S1.

\section{Construction of the LmjF.22.0600-overexpressing strain}

For the construction of the LmjF.22.0600-overexpressing strain, LmjF.22.0600 was PCR-amplified from $L$. major genomic DNA. The PCR product was cloned into pGEM-T-Easy (Promega, Madison, WI, USA) and then inserted into the expression vector pTH6nGFPc (kind gift from Patrick Bastien, Montpellier University) after digestion with MfeI and HpaI restriction enzymes, vector that places the GFP sequence at the 3'-end of the LmjF.22.0600 sequence. The primers used are listed in Additional file 4: Table S1.

Deletion of the LmjF.22.0600 gene was performed as described in the article of Beneke et al. [28]. The primers were designed thanks to the online tool developed by the authors: http://leishgedit.net/ (see Additional file 4: Table S1). For the PCR-amplification of the donor DNA, $20 \mathrm{ng}$ of plasmids LC100 and LC101 (kind gift from Eva Gluenz, University of Oxford), $0.4 \mathrm{mM}$ dNTP, $0.5 \mu \mathrm{M}$ each of gene-specific forward and reverse primers and $0.5 \mu \mathrm{l}$ of Phusion High-Fidelity DNA Polymerase (New England Biolabs, Ipswich, MA, USA) were mixed in a final volume of $50 \mu \mathrm{l}$. To amplify the guide RNAs, $0.6 \mathrm{mM}$ $\mathrm{dNTP}, 2 \mu \mathrm{M}$ each of gene-specific forward and reverse primers and $0.5 \mu \mathrm{l}$ of Phusion High-Fidelity DNA Polymerase were mixed in a final volume of $50 \mu \mathrm{l}$. The PCR conditions were as follows: $30 \mathrm{~s}$ at $98{ }^{\circ} \mathrm{C}$, then 40 cycles of $10 \mathrm{~s}$ at $98{ }^{\circ} \mathrm{C}, 30 \mathrm{~s}$ at $62{ }^{\circ} \mathrm{C}$ and $1 \mathrm{~min}$ at $72{ }^{\circ} \mathrm{C}$, with a final elongation step of $10 \mathrm{~min}$ at $72{ }^{\circ} \mathrm{C}$. After assessing the presence of the expected PCR products by migration on an agarose gel, the PCR products (each PCR was done in duplicate) were pooled and purified with the Wizard SV Gel and PCR Clean-Up System (Promega). The purified PCR products were heat sterilized at $95^{\circ} \mathrm{C}$ for $5 \mathrm{~min}$ prior to transfection. 


\section{Transfection procedure}

For the LmjF.22.0600 overexpression, $3 \times 10^{7}$ L. major cells in logarithmic phase were transfected with $10 \mu \mathrm{g}$ of the recombinant vector pTH6nGFPc containing the LmjF.22.0600 sequence, in $100 \mu \mathrm{l}$ of Human T Cell Nucleofector solution (Lonza, Basel, Switzerland).

For the LmjF.22.0600 deletion, $10^{7}$ L. major cells expressing Cas9 and T7 (kind gift from Eva Gluenz, University of Oxford) in logarithmic phase were transfected with $30 \mu \mathrm{g}$ of purified PCR. The transfection buffer was composed of $90 \mathrm{mM}$ sodium phosphate, $5 \mathrm{mM}$ potassium chloride, $0.15 \mathrm{mM}$ calcium chloride, $50 \mathrm{mM}$ HEPES, pH 7.3. The same transfection without purified PCR was used as control.

The transfections were performed in $2 \mathrm{~mm}$ gap cuvettes (Lonza) with program X-001 of the Amaxa Nucleofector II (Lonza). Transfected cells were immediately transferred into $5 \mathrm{ml}$ pre-warmed medium and left to recover overnight at $26{ }^{\circ} \mathrm{C}$ before adding $30 \mu \mathrm{g} / \mathrm{ml}$ hygromycin $\mathrm{B}$ (Thermo Fisher Scientific) for the overexpression and two drugs for the deletion: geneticin (Sigma-Aldrich) at 20 $\mu \mathrm{g} / \mathrm{ml}$ and puromycin (Sigma-Aldrich) at $30 \mu \mathrm{g} / \mathrm{ml}$.

\section{MTT assay}

WT L. major cells or cells overexpressing LmjF.22.0600 were incubated in triplicate at $10^{6}$ cells $/ \mathrm{ml}$ with various concentrations of curcumin, miltefosine or pentamidine for $72 \mathrm{~h}$. As controls, cells were also incubated with ethanol (curcumin solvent) and $\mathrm{H}_{2} \mathrm{O}$ (miltefosine and pentamidine solvent). MTT at $5 \mathrm{mg} / \mathrm{ml}(20 \mu \mathrm{l})$ was then added. Cells were further incubated for $4 \mathrm{~h}$ at $26^{\circ} \mathrm{C}$ and $100 \mu \mathrm{l}$ of a blocking solution was added $(10 \% \mathrm{~m} / \mathrm{v}$ sodium dodecyl sulfate and $50 \% \mathrm{v} / \mathrm{v}$ isopropanol). Optical density, measured with a spectrophotometer (ELX808 Ultra Microplate Reader; Bio-Tek Instruments, Colmar, France) at $570 \mathrm{~nm}$, allowed us to determine the drug IC50, the drug concentration inhibiting $50 \%$ of cell growth compared to the control.

\section{TUNEL assay}

The In Situ Cell Death Detection Kit, Fluorescein (Roche), was used for detecting DNA fragmentation characteristics of apoptotic cells by fluorescence microscopy. To do so, cells were fixed with formaldehyde $4 \%$, laid on an immunoslide and permeabilized with a $0.1 \%$ triton and $0.1 \%$ sodium citrate solution. The reaction solution from the kit, with the enzyme diluted $1 / 10$, was then added, before observation with a BX51 fluorescence microscope (Olympus, Rungis, France). Bright field and fluorescence images were acquired using the fluorescence imaging system Cell $^{\mathrm{A}}$ (Olympus).

\section{Calcein and PI labeling}

Cells were washed once in PBS and resuspended in $1 \mathrm{ml}$ of PBS containing $2 \mu \mathrm{l}$ of calcein (LIVE/DEAD ${ }^{\circledR}$ Viability/Cytotoxicity Kit for mammalian cells; Molecular Probes, Eugene, OR, USA) diluted 1/80 in DMSO and $5 \mu \mathrm{l}$ of PI at $0.5 \mathrm{mg} / \mathrm{ml}$. The mixed sample was then incubated for $15-20 \mathrm{~min}$ at room temperature and protected from light. The cells were analyzed by flow cytometry using an excitation wavelength of $488 \mathrm{~nm}$ and measuring green fluorescence emission for calcein (530/30 nm bandpass) and red fluorescence emission for PI (610/20 $\mathrm{nm}$ bandpass) on a BD LSRFortessa ${ }^{\mathrm{TM}}$ cell analyzer (BD, Le Pont de Claix, France). Data were exported and analyzed with Flowjo software for evaluation of the percentage of calcein- and PI-positive cells.

\section{Statistical analysis}

For statistical analysis, unpaired Wilcoxon-MannWhitney tests or t-tests were performed with BioStaTGV (https://biostatgv.sentiweb.fr/?module=tests). Results, obtained from a minimum of three independent experiments, were considered statistically significant when $P<0.05$; otherwise, "ns" was written, for not significant. For significant differences: ${ }^{*} P<0.05$, ${ }^{* *} P<$ 0.01 and ${ }^{* * *} P<0.001$.

\section{Additional files}

Additional file 1: Figure S1. The overexpression of LmjF.22.0600 induces no growth defect. Growth curves of the WT and LmjF.22.0600-overexpressing [WT(LmjF.22.0600)] cells: $n \geq 3$.

Additional file 2: Figure S2. The LmjF.22.0600 gene is present in WT and LmjMCA deleted cells. Agarose gel electrophoresis after a PCR with LmjF.22.0600-specific primers, showing the presence of the LmjF.22.0600 gene in the WT cell line as well as in the LmjMCA-deleted cells ( $\triangle$ LmjMCA)

Additional file 3: Figure S3. The inhibition of $L m j F .22 .0600$ expression by CRISPR/Cas9 induces almost no change concerning L. major apoptosis. a Cell concentration of WT and LmjF.22.0600-deleted cells after curcumin (30 $\mu \mathrm{M})$, miltefosine $(40 \mu \mathrm{M})$ and pentamidine $(100 \mu \mathrm{M})$ induced cell death, as measured with a hemocytometer. Values are shown as the mean \pm $S D$. The number of independent experiments is written in the figure. $\mathbf{b}$ Percentage of calcein-positive WT and LmjF.22.0600-deleted cells after the induction of $L$. major apoptosis with $30 \mu \mathrm{M}$ curcumin, $40 \mu \mathrm{M}$ miltefosine or $100 \mu \mathrm{M}$ pentamidine. No significant difference was observed between the WT and the deleted strains, according to a Mann-Whitney test. c Percentage of PI-positive WT and LmjF.22.0600-deleted cells after the induction of L. major apoptosis with $30 \mu \mathrm{M}$ curcumin, $40 \mu \mathrm{M}$ miltefosine or $100 \mu \mathrm{M}$ pentamidine. No significant difference was observed between the WT and the deleted strains, according to a Mann-Whitney test.

Additional file 4: Table S1. Oligonucleotides used for RT-qPCR, LmjF.22.0600 overexpression and LmjF.22.0600 deletion. 


\section{Abbreviations}

GFP: green fluorescent protein; IC50: inhibitory concentration 50\%; MCA: metacaspase; MTT: methyl thiazol tetrazolium; PI: propidium iodide; RT-qPCR: reverse-transcription quantitative $P C R$.

\section{Acknowledgments}

We want to thank Eva Gluenz (Oxford University) for the Cas9/T7 L. major expressing strain and for the pT plasmids for CRISPR/Cas9 constructions, as well as Patrick Bastien from Montpellier University, for the pTH6nGFPc expression vector, and the CNR Leishmania (Montpellier, France) for the L. major strain.

\section{Authors' contributions}

LB analyzed the TUNEL data and revised the manuscript. NA provided funding MC conceived the study, performed the experiments, analyzed the results and wrote the paper. All authors read and approved the final manuscript.

\section{Funding}

This study was supported by the Institut Hospitalo-Universitaire (IHU) Méditerranée Infection, the National Research Agency under the program "Investissements d'avenir", reference ANR-10-IAHU-03, the Région Provence-Alpes-Côte d'Azur and European funding FEDER PRIMI.

\section{Availability of data and materials}

All data generated or analyzed during this study are provided within the article and its additional files or are available from the corresponding author upon request.

\section{Ethics approval and consent to participate}

Not applicable.

\section{Consent for publication}

Not applicable.

\section{Competing interests}

The authors declare that they have no competing interests.

\section{Author details}

1 UMR PAM A, Valmis team, 2 rue Angélique Ducoudray, BP 37013, 21070 Dijon Cedex, France. ${ }^{2}$ Aix Marseille Univ, IRD, AP-HM, SSA, VITROME, Marseille, France. ${ }^{3}$ IHU-Méditerranée Infection, Marseille, France.

Received: 18 December 2018 Accepted: 23 May 2019

Published online: 27 May 2019

\section{References}

1. Kroemer G, Galluzzi L, Vandenabeele P, Abrams J, Alnemri ES, Baehrecke $\mathrm{EH}$, et al. Classification of cell death: recommendations of the Nomenclature Committee on Cell Death 2009. Cell Death Differ. 2009;16:3-11.

2. Jiménez-Ruiz A, Alzate JF, Macleod ET, Lüder CGK, Fasel N, Hurd H. Apoptotic markers in protozoan parasites. Parasit Vectors. 2010;3:104.

3. Proto WR, Coombs GH, Mottram JC. Cell death in parasitic protozoa: regulated or incidental? Nat Rev Microbiol. 2013;11:58-66.

4. Casanova M, Gonzalez IJ, Sprissler C, Zalila H, Dacher M, Basmaciyan L, et al. Implication of different domains of the Leishmania major metacaspase in cell death and autophagy. Cell Death Dis. 2015;6:e1933.

5. Castanys-Muñoz E, Brown E, Coombs GH, Mottram JC. Leishmania mexicana metacaspase is a negative regulator of amastigote proliferation in mammalian cells. Cell Death Dis. 2012;3:e385.

6. Alcolea PJ, Alonso A, Gomez MJ, Sanchez-Gorostiaga A, Moreno-Paz M,

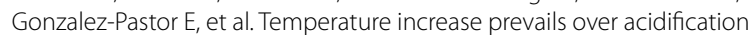
in gene expression modulation of amastigote differentiation in Leishmania infantum. BMC Genomics. 2010;11:31.

7. Bente M, Harder S, Wiesgigl M, Heukeshoven J, Gelhaus C, Krause E, et al. Developmentally induced changes of the proteome in the protozoan parasite Leishmania donovani. Proteomics. 2003:3:1811-29.
8. Biyani N, Madhubala R. Quantitative proteomic profiling of the promastigotes and the intracellular amastigotes of Leishmania donovani isolates identifies novel proteins having a role in Leishmania differentiation and intracellular survival. Biochim Biophys Acta. 2012;1824:1342-50.

9. McNicoll F, Drummelsmith J, Müller M, Madore É, Boilard N, Ouellette M, et al. A combined proteomic and transcriptomic approach to the study of stage differentiation in Leishmania infantum. Proteomics. 2006;6:3567-81.

10. Nugent PG, Karsani SA, Wait R, Tempero J, Smith DF. Proteomic analysis of Leishmania mexicana differentiation. Mol Biochem Parasitol. 2004;136:51-62.

11. Rosenzweig D, Smith D, Myler PJ, Olafson RW, Zilberstein D. Post-translational modification of cellular proteins during Leishmania donovani differentiation. Proteomics. 2008;8:1843-50.

12. Walker J, Vasquez J-J, Gomez MA, Drummelsmith J, Burchmore R, Girard I, et al. Identification of developmentally-regulated proteins in Leishmania panamensis by proteome profiling of promastigotes and axenic amastigotes. Mol Biochem Parasitol. 2006;147:64-73.

13. Besteiro S, Williams RAM, Coombs GH, Mottram JC. Protein turnover and differentiation in Leishmania. Int J Parasitol. 2007;37:1063-75.

14. Dagger F, Bengio C, Martinez A, Ayesta C. Leishmania mexicana differentiation involves a selective plasma membrane autophagic-like process. Cell Stress Chaperones. 2018;23:783-9.

15. Mariño G, Niso-Santano M, Baehrecke EH, Kroemer G. Self-consumption: the interplay of autophagy and apoptosis. Nat Rev Mol Cell Biol. 2014;15:81-94.

16. Basmaciyan L, Berry L, Gros J, Azas N, Casanova M. Temporal analysis of the autophagic and apoptotic phenotypes in Leishmania parasites. Microb Cell. 2018;5:404-17.

17. Basmaciyan L, Azas N, Casanova M. Different apoptosis pathways in Leishmania parasites. Cell Death Discov. 2018;5:27.

18. Basmaciyan L, Azas N, Casanova M. Calcein+/PI- as an early apoptotic feature in Leishmania. PLoS ONE. 2017;12:e0187756.

19. Gannavaram S, Debrabant A. Programmed cell death in Leishmania: biochemical evidence and role in parasite infectivity. Front Cell Infect Microbiol. 2012;2:95.

20. Shaha C. Apoptosis in Leishmania species \& its relevance to disease pathogenesis. Indian J Med Res. 2006;123:233-44.

21. Gannavaram S, Vedvyas C, Debrabant A. Conservation of the pro-apoptotic nuclease activity of endonuclease $\mathrm{G}$ in unicellular trypanosomatid parasites. J Cell Sci. 2008;121:99-109.

22. Rico E, Alzate JF, Arias AA, Moreno D, Clos J, Gago F, et al. Leishmania infantum expresses a mitochondrial nuclease homologous to EndoG that migrates to the nucleus in response to an apoptotic stimulus. Mol Biochem Parasitol. 2009;163:28-38.

23. El-Fadili AK, Zangger H, Desponds C, Gonzalez IJ, Zalila H, Schaff C, et al. Cathepsin B-like and cell death in the unicellular human pathogen Leishmania. Cell Death Dis. 2010;1:e71.

24. Genes CM, de Lucio H, González VM, Sánchez-Murcia PA, Rico E, Gago F, et al. A functional $\mathrm{BH} 3$ domain in an aquaporin from Leishmania infantum. Cell Death Discov. 2016;2:16043.

25. Basmaciyan $L$, Jacquet $P$, Azas N, Casanova M. A novel hydrolase with a pro-death activity from the protozoan parasite Leishmania major. Cell Death Discov. 2019 (In press)

26. Bröker LE, Kruyt FAE, Giaccone G. Cell death independent of caspases: a review. Clin Cancer Res. 2005;11:3155-62.

27. Pfaffl MW. A new mathematical model for relative quantification in realtime RT-PCR. Nucleic Acids Res. 2001;29:e45.

28. Beneke T, Madden R, Makin L, Valli J, Sunter J, Gluenz E. A CRISPR Cas 9 high-throughput genome editing toolkit for kinetoplastids. R Soc Open Sci. 2017:4:170095.

\section{Publisher's Note}

Springer Nature remains neutral with regard to jurisdictional claims in published maps and institutional affiliations. 